percentage of (1) plus (2) to the total number of students :

\begin{tabular}{|c|c|c|c|c|}
\hline & & \multicolumn{2}{|c|}{ Students from abroad } & $\begin{array}{c}\text { (Per cent) } \\
(3)\end{array}$ \\
\hline London & $\ldots$ & 1,191 & 674 & 16 \\
\hline Sch. of Eco & aies & 141 & 234 & 39 \\
\hline Imperial Cc & & 163 & 50 & 20 \\
\hline University & $\ldots$ & 221 & 118 & 17 \\
\hline Guy's Hosp & & 99 & 18 & 14 \\
\hline St. Bart.'s & spl. & 78 & 34 & 15 \\
\hline Oxford & . & 290 & 234 & 11 \\
\hline Cambridge & . & 346 & 192 & 9 \\
\hline Birmingham & . & 28 & 85 & 11 \\
\hline Edinburgh & $\ldots$ & 329 & 176 & 14 \\
\hline St. Andrews & $\ldots$ & 14 & 87 & 11 \\
\hline
\end{tabular}

In view of current controversies and world-wide perplexity in regard to economic doctrine, it is noteworthy that the London School of Economies draws so many students from abroad.

Analysis according to subjects of study reveals a substantial increase of 807 (eight per cent) in the number of medical students and a falling off of five per cent under agriculture.

University finances, which are to a large extent dominated by State aid-parliamentary and local authorities' grants together amounting to $44 \frac{1}{2}$ per cent of the aggregate of university incomes-have called for close and unremitting attention on the part of university administrative authorities since the national financial crisis of 1931. Evidence of the effectiveness of their control is to be found in the fact that there were only four institutions the accounts of which showed deficits on the year and in only one was the deficit of any substantial amount. The aggregate amount of debt on capital account at the close of the year was about one million pounds, or one sixth of the aggregate annual income.

Expenditure on libraries is dissected in a special table which shows that of the total, $£ 210,756$, sixteen per cent was on account of purchase of periodicals, the increasing number and cost of which was already, three years ago, when they were responsible for little more than ten per cent of library expenditure, a source of embarrassment.

Four London institutions now make their first appearance in the refurns: the Courtauld Institute of Art, first opened in October, 1932 ; the Institute of Education (formerly the London Day Training College), transferred from the control of the London County Council to that of the University of London in September, 1932; the Institute of Historical Research, statistics of which were previously not differentiated in the returns from those of the University of London as a whole; and the School of Slavonic and East European Studies, transferred from King's College to the University of London in August 1932.

\title{
Quantitative Methods of Biological Assay
}

\section{$\mathrm{T}$} HERAPEUTIC substances, which cannot yet be completely defined by their physical and chemical characteristics, can only be used with safety when their activity has been determined by tests on animals. The accuracy of such tests has been greatly increased during the last decade, especially since it was realised that large numbers of animals must be used in each assay, so that the average response of a group of animals can be determined, allowance thus being made for the differences in response of the individual animals. Such tests may be of two types : either the response of each animal to the drug is accurately measured, for example, the effect of insulin upon the blood sugar of the rabbit; or observation is only made as to whether some specific effect is produced or not, for example, the effect of insulin upon the mouse, when the end-point is the occurrence, or not, of convulsions. Gaddum has recently made an analysis of the latter type of test and his report should be of great value in the interpretation of the results of such experiments.*

In tests of the 'all-or-none' or 'quantal' type, it is now usual to inject several doses of the substance under test into a series of animals, ten or twenty or more being used for each dose, and to plot the percentage number responding on each dose against the dose given. The curve which is then drawn to pass approximately through the plotted points is $S$-shaped, the shape and slope of the curve being characteristic for each combination of drug and test-animal species. It is often more convenient to plot the effect of the drug against the logarithm of the dose (to base 10) instead of against the dose itself ; the characteristic curves are then easier to interpret. The most satis-

* Reports on Biological Standards. (3) "Methods of Biological Assay depending on a Quantal Response." By J. H. Gaddum. Stationery Office, London, 1933.) 18. net. factory index of the slope of the curve, and so of the uniformity of the animals, is the standard deviation of the logarithms of the individual effective doses, which can be estimated with sufficient accuracy by taking from the curve the log-dose corresponding to 84 per cent, subtracting from it the log-dose corresponding to 16 per cent and halving the result.

Gaddum also recommends that instead of using the percentage of responses on each dose as a measure of the effect, the normal equivalent deviation be plotted against the logarithm of the dose, since this function gives a more satisfactory measure of the effect than the percentage does. It is equal to the deviation from the mean, and is obtained in practice from tables. When the normal equivalent deviations are plotted against the logarithms of the doses, the points so obtained lie approximately on straight lines, when the logarithms of the individual effective doses are normally distributed. It is therefore usually sufficient to use only two doses and to take the line joining them as an indication of the relation between the logarithm of the dose and the effect. This line is completely described by calculating the dose which produces the effect in half the animals and the standard deviation of the logarithms of the individual effective doses.

The report is illustrated by a number of curves obtained by different observers in assays of different drugs or hormones such as ouabain, neoarsphenamine, œstrin and pneumococcus antibody. The control of variables which affect the homogeneity of the animals used, such as their genetic composition, weight, age, sex, diet and environmental temperature, is also discussed. The mathematical argument involved in the use of these curves is described and, in greater detail, the methods of carrying out a test, two examples being included. 